Epidemiology and Infection

cambridge.org/hyg

\section{Original Paper}

Cite this article: Mukanyangezi MF, Manzi O, Tobin G, Rulisa S, Bienvenu E, Giglio D (2019). Sexual risk behaviour in a cohort of HIV-negative and HIV-positive Rwandan women. Epidemiology and Infection 147, e54, 1-9. https://doi.org/10.1017/ S0950268818003023

Received: 27 May 2018

Revised: 28 August 2018

Accepted: 10 October 2018

\section{Key words:}

Candida; gonorrhoea; HIV/AIDS; infectious disease epidemiology; sexually transmitted infections (STIs)

Author for correspondence: D. Giglio, E-mail: daniel.giglio@pharm.gu.se

\title{
Sexual risk behaviour in a cohort of HIV-negative and HIV-positive Rwandan women
}

M. F. Mukanyangezi ${ }^{1,2}$, O. Manzi ${ }^{3}$, G. Tobin ${ }^{1}$, S. Rulisa ${ }^{3,2}$, E. Bienvenu ${ }^{2}$

and D. Giglio ${ }^{1,4}$

${ }^{1}$ Department of Pharmacology, University of Gothenburg, Gothenburg, Sweden; ${ }^{2}$ College of Medicine and Health Sciences, University of Rwanda, Kigali, Rwanda; ${ }^{3}$ University Teaching Hospital of Kigali (CHUK), Kigali, Rwanda and ${ }^{4}$ Department of Oncology, University of Gothenburg, Gothenburg, Sweden

\begin{abstract}
Here we wanted to assess whether sexual risk behaviour differs dependent by human immunodeficiency virus (HIV) status by following $100 \mathrm{HIV}-$ and $137 \mathrm{HIV}+$ women recruited at two university teaching hospitals in Rwanda. Women were tested for sexually transmitted infections (STIs; trichomoniasis, syphilis, hepatitis B and C) and for reproductive tract infections (RTIs; candidiasis, bacterial vaginosis (BV)) and were interviewed at baseline and 9 months later. BV was the most prevalent infection, while syphilis was the most common STI with a 9 -month incidence of $10.9 \%$ in HIV+ women. Only $24.5 \%$ of women positive for any RTI/STI contacted their health facility and got treatment. More HIV- women than HIV+ women had had more than one sexual partner and never used condoms during the follow-up period. The use of condoms was affected neither by marital status nor by concomitant STIs besides HIV. Our data highlight the importance of public education regarding condom use to protect against STIs in an era when HIV no longer is a death sentence.
\end{abstract}

\section{Introduction}

Transmission of sexually transmitted infections (STIs) is a serious health care problem in many countries in Africa [1-3]. STIs such as trichomoniasis and syphilis and other reproductive tract infections (RTIs) such as bacterial vaginosis (BV) and candidiasis have been shown to increase the risk of acquiring human immunodeficiency virus (HIV) infection [4-7]. HIV infection may on the other hand increase the risk of acquiring STIs including human papillomavirus (HPV) [8], syphilis [9], candidiasis [10], herpes [11] and genital ulcer disease [10]. Changes in the microenvironment of the vagina such as in BV may increase the risk of contracting STIs especially among HIV-infected women [12]. Studies on the prevalence and character of STIs and RTIs in Rwanda are only a few. A cross-sectional nationwide survey in Rwanda showed a prevalence of syphilis of $0.8 \%$ among HIV-negative and $4.8 \%$ among HIV-positive women [13]. In our previous study, we showed that HPV infections and other STIs and RTIs are very common in Rwandan women [14].

Rwanda has taken measures to prevent the spread and consequences of STIs. Among the most important interventions are that the World Health Organization (WHO) recommended couples' voluntary HIV counselling and testing has been implemented, that all patients diagnosed with HIV are offered antiretroviral therapy and school girls are offered HPV vaccine [15]. However, in view of the high degree of STIs in the country, it is important to understand the underlying mechanisms leading to the spread of STIs. In the present study, we assessed whether concomitant HIV infection changes the chance of clearing STIs and RTIs in Rwanda. We also assessed attitudes to STIs and RTIs and sexual risk behaviour among Rwandan women. This study shows that there is a high turnover of STIs in Rwanda and that present STIs may not necessarily change sexual risk behaviour.

\section{Methods}

Women recruited in the present study consist of a subset of those described in our previous study [14]. Adult women were recruited at the University Teaching Hospitals in Kigali (CHUK) and Butare (CHUB) in Rwanda. Written informed consent was obtained from all subjects before enrolment. Exclusion criteria were pregnancy, being diagnosed with HPV infection before enrolment in the present study and/or cervical cancer, the presence of any known disease likely to limit life expectancy to $<24$ months and presenting any other factor suggesting inability to comply with the study protocol. Socio-demographic data were collected by interviewing women and included sexual, behaviour and obstetric, gynaecological and STI 
history. In the present study, 119 women with cervical cytological abnormalities and/or HPV positivity with an age-matched 118 cohort of healthy controls were selected to constitute the two cohorts for a longitudinal study. Of the 237 women, 58\% were HIV-positive and 42\% HIV-negative. Women were tested for trichomoniasis, syphilis, HPV, vaginal candidiasis, BV, hepatitis $\mathrm{B}$, hepatitis $\mathrm{C}$ and HIV at inclusion. Women were called back 9 months later for a new interview, physical examination and a repeated laboratory testing. All laboratory results for STIs/RTIs were reported back to women within 2 weeks after testing through a telephone call. Women were instructed to visit a health care facility if being positive for any treatable STI/RTI. The Institutional Review Board, College of Medicine and Health Sciences, at the University of Rwanda and the Ethics Committee at the University of Gothenburg, Gothenburg, Sweden, approved the study.

\section{HIV testing}

A well-trained laboratory technician performed HIV rapid testing in accordance with national HIV rapid test algorithm. Women were first screened by the use of the Alere HIV Combo (Alere Medical Co., Ltd, Chiba, Japan), and then reactive samples were confirmed by the second test, i.e. HIV $1 / 2$ Stat-Pak ${ }^{\otimes}$ (Chembio Diagnostic System, Inc., Medford, NY, USA). Reactive samples were finally reported as positives. Cluster of differentiation (CD4) count and viral load data of HIV+ women were extracted from clinical records.

\section{HPV testing}

The Multiplex Luminex system (Bio-Rad Laboratories, Inc., Irvine, CA, USA) was used to detect the following HPV strains: low-risk (LR)-HPV: 6, 11, 30, 40, 42, 43, 54, 61, 67, 73, 74, 81, $82,83,86,87,89,90,91$; high-risk (HR)-HPV: 16, 18, 18v, 31, $33,35,35 \mathrm{v}, 39,45,51,52,56,58,59$; and possibly high-risk (PHR)-HPV 26, 53, 66, 68a, 68b, 69, 70 [16, 17], according to manufacturer's instructions.

\section{Testing for hepatitis $B$ and $C$}

Screening for hepatitis B was performed using the hepatitis B virus surface antigen (HBsAg) Rapid Test Strip (Rapid Labs Ltd, Colchester, UK) to detect HBsAg. Hepatitis C testing was performed using the hepatitis C Rapid Test Strip (Rapid Labs Ltd). This test detects hepatitis $C$ virus antibody.

\section{Testing for other STIS}

The vaginal swab was placed in $0.2 \mathrm{ml}$ of sterile physiologic saline for wet mount evaluation and then examined microscopically within $15 \mathrm{~min}$ for motile Trichomonas vaginalis. It was also used for vaginal candidiasis testing. Testing for syphilis was conducted using the rapid plasma reagin (RPR) test (BD Macro-Vue RPR Card Test, BD, USA; Franklin Lakes, NY, USA). The RPR-positive samples were sent to the National Reference Laboratory within $24 \mathrm{~h}$ for confirmation using the Treponema pallidum haemagglutination test, the rapid test to detect syphilis antibodies. A sample that was positive for both tests was recorded as positive and a non-reacting sample to the second test was recorded as negative.

\section{Testing for RTIS}

Cervical-vaginal samples were cultured on Sabouraud culture medium for the detection of candidiasis. The identity of clinical isolates was confirmed by germ tube induction test in serum for Candida albicans. The presence of BV was evaluated microscopically on samples collected from the posterior vaginal fornix. BV was diagnosed by the presence of three of the clinical and microscopic findings standardised by the Nugent scoring method [18].

\section{Statistical analysis}

IBM SPSS statistics version 22 and Graph Pad 7 software were used for data analysis. Syphilis, trichomoniasis, hepatitis B and hepatitis $\mathrm{C}$ were considered as STIs, while BV and candidiasis were considered as RTIs. Since the number of individual RTI and STI cases was relatively low in the studied cohort, statistics were performed on the STI and RTI groups. By the same reason, PHR and HR-HPV infections were combined in one category denoted HR-HPV. Mean \pm standard deviation and proportion were computed for descriptive analysis. Depending on the sample size, the $\chi^{2}$ test or the Fisher's exact test was used to compare groups. To identify basal characteristics and prevalence of STIs/ RTIs at 9 months, univariate logistic regression analysis was performed and odds ratios (ORs) with 95\% confidence intervals (CIs) were calculated. Results were considered as statistically significant at $P<0.05$.

\section{Results}

Demographics, sexual and clinical characteristics at baseline

The demographic data are displayed in Table 1 and resemble the data from our previous study on the larger cohorts of women [14]. The mean age of women was $44 \pm 8$ years ( $41 \pm 8$ and $47 \pm 8$ years for HIV - and HIV+ women, respectively) and $80.6 \%$ of women were 35 years old or older. The majority $(68.4 \%)$ had only primary education or less (1-11 years of schooling). More HIVthan HIV+ women, $(68.0 \%$ vs. $28.5 \%$, respectively; $P<0.001)$ were married. It was more common among HIV+ women than among HIV - women to have had the sexual debut before the age of 21 (reported by $73.0 \%$ and $48.0 \%$, respectively; $P<$ 0.001 ), to have had more than one lifetime sexual partner (reported by $73.7 \%$ and $38.0 \%$, respectively; $P<0.001$ ) and being infected previously by gonorrhoea $(21.2 \%$ and $6.0 \%$, respectively; $P<0.001$; Table 1 ). More HIV+ than HIVwomen claimed not knowing if they had been infected previously by chlamydia $(29.2 \%$ and $4.0 \%$; $P<0.001$; Table 1$)$. The majority of HIV+ women had a CD4 count over 200 cells/ $\mu$ with a viral load of $<400$ copies (Table 1 ).

\section{Prevalence, incidence and persistence of STIs and RTIS}

In Tables 2 and 3, the prevalence, incidence and persistence of STIs and RTIs in HIV - and HIV+ women are described. At baseline, the prevalence of being diagnosed with one or more STIs/ RTIs was higher in HIV - women than in HIV+ women $(66.0 \%$ vs. $26.2 \%$, respectively; $P<0.001$ ); however, at 9 months no difference in prevalence of STIs/RTIs between HIV- and HIVwomen could be observed $(40.0 \%$ and $46.0 \%$, respectively, Table 2). The prevalence of syphilis at baseline and 9 months was $1.4 \%$ and $0 \%$ in HIV - women and $0.8 \%$ and $10.9 \%$ in $\mathrm{HIV}+$ women, respectively. The 9 -month incidence was $0 \%$ in 
Table 1. Demographic data, sexual and biological characteristics among HIV- and HIV+ women

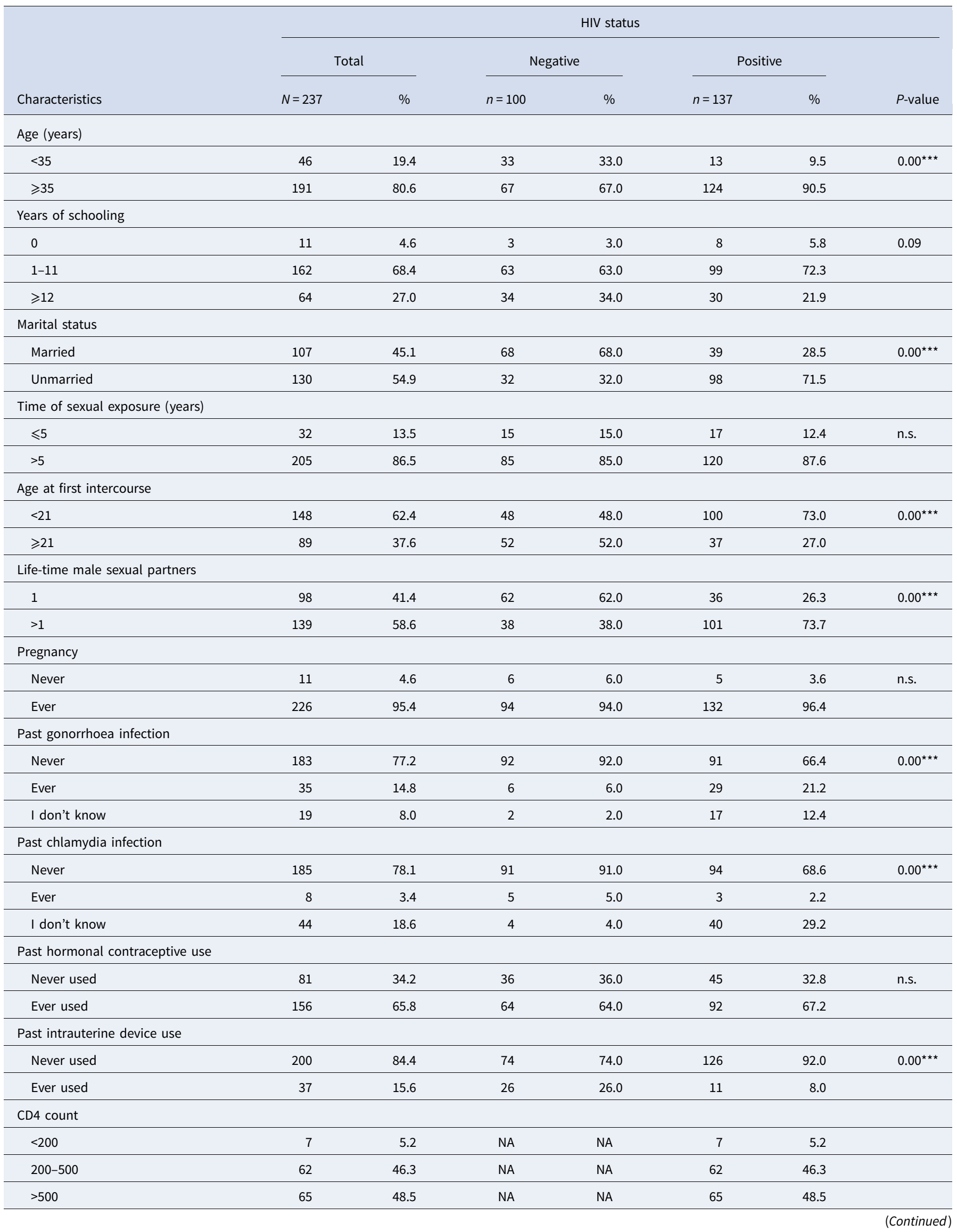


Table 1. (Continued.)

\begin{tabular}{|c|c|c|c|c|c|c|c|}
\hline \multirow[b]{3}{*}{ Characteristics } & \multicolumn{7}{|c|}{ HIV status } \\
\hline & \multicolumn{2}{|c|}{ Total } & \multicolumn{2}{|c|}{ Negative } & \multicolumn{2}{|c|}{ Positive } & \multirow[b]{2}{*}{$P$-value } \\
\hline & $N=237$ & $\%$ & $n=100$ & $\%$ & $n=137$ & $\%$ & \\
\hline \multicolumn{8}{|l|}{ Viral load } \\
\hline$<400$ & 117 & 87.3 & NA & NA & 117 & 87.3 & \\
\hline $400-5000$ & 7 & 5.2 & NA & NA & 7 & 5.2 & \\
\hline$>5000$ & 10 & 7.5 & NA & NA & 10 & 7.5 & \\
\hline
\end{tabular}

The $\chi^{2}$ test was used to compare factors between HIV- and HIV+ women. ${ }^{\star} P<0.05$ and ${ }^{\star \star \star} P<0.001$. NA, not applicable.

HIV - women and $10.9 \%$ in HIV+ women $(P<0.01$; Table 3$)$. The prevalence of candidiasis at baseline and 9 months was $12 \%$ and $24 \%$ in HIV - women and $9.5 \%$ and $14.6 \%$ in HIV+ women, respectively. Persistent candidiasis was developed in $50 \%$ of HIV - women and in $7.7 \%$ of HIV+ women $(P<0.05$; Table 3). While only one out of 12 infections were treated in the HIV - group, four out of 13 infections were treated in the $\mathrm{HIV}+$ group. Prevalence of hepatitis B was $2.3-4.7 \%$ in HIVwomen and $1.6-3.1 \%$ in HIV+ women, prevalence of hepatitis C was $0 \%$ in HIV - women and $3.1-4.7 \%$ in HIV+ women, prevalence of trichomoniasis was $3.0-5.1 \%$ in HIV- women and 2.9$3.6 \%$ in HIV+ women and prevalence of BV was $15.0-18.0 \%$ in HIV- women and $8.8-22.6 \%$ in HIV+ women. There were no significant differences between HIV - and HIV+ women in the prevalence of hepatitis $\mathrm{B}$, hepatitis $\mathrm{C}$, trichomoniasis and BV. Figure S1 displays the distribution of STIs/RTIs at 9 months between HIV - and HIV+ women stratified according to the absence or presence of concomitant LR- or HR-HPV infections at baseline. STIs and RTIs were similarly distributed among women regardless of co-infection of HPV. No correlation between baseline characteristics and prevalence of STIs/RTIs at 9 months was observed (Table S1).

\section{Treatment of STIS/RTIS}

The interview conducted at the 9 months follow-up revealed that among women who were tested positive for any STI/RTI at baseline, only $13 / 53$ women $(24.5 \%)$ contacted their health facilities and got treatment. The percentages of patients seeking treatment and curing rates of STIs/RTIs are summarised in Figure 1a. HIV+ women tended to seek treatment more often than HIV- women ( $36.4 \%$ and $16.1 \%$, respectively; $P=0.11$ ). The number of patients treated for STIs/RTIs was low in both studied cohorts giving low power to the statistical analysis. When considering all STIs/RTIs combined, $25 \%$ of HIV+ women were cured by treatment compared with $80 \%$ of HIV- women (n.s.). In HIV- women, 13/ 26 STIs/RTIs (50\%), and in HIV+ women, 9/14 STIs/RTIs (64\%) were resolved spontaneously (n.s.).

\section{Time-varying behaviour characteristics among women during follow-up period}

Seventy-five per cent of HIV- women and 35\% of HIV+ women reported to have had sex during the 9 months follow-up. The majority $(87 \%)$ reported to have had only one sexual partner and $41 \%$ never used condom for protection. More HIVwomen had had more than one sexual partner than HIV+ women during the follow-up period ( $20 \%$ vs. $2.1 \%$, respectively; $P<0.001)$. More HIV - women than HIV+ women never used condom during the follow-up $(57.3 \%$ vs. $14.6 \%$, respectively; $P<0.001$, Fig. 1b). Among HIV+ women, 10 women reported not having sex but still contracted syphilis during the follow-up.

\section{Effect of HIV and STIs testing at baseline on sexual behaviour}

Marital status did not affect the degree of condom use, i.e. while all unmarried HIV+ women reported using condoms, as well as the majority of all married HIV+ women $(82 \%)$, the corresponding figures for unmarried and married HIV- women were $30 \%$ and $45 \%$, respectively (Fig. $2 \mathrm{a}$ ). In both groups, there was no significant difference in condom use between married and unmarried women. Furthermore, no change in attitudes to condom use was observed among women detected with STIs at baseline. Only one HIV+ woman was positive for STI and she reported not having used condom during sexual intercourse during the 9-month follow-up. Almost all STI-positive HIV- women $(87.5 \%)$ reported not to have used condoms during sexual intercourse during the 9-month follow-up (Fig. 2b).

\section{Discussion}

We currently examined the effect of testing for HIV and STIs/ RTIs on future sexual risk behaviour in a cohort of HIV- and $\mathrm{HIV}+$ Rwandan women. The present study shows a high use of condoms among HIV+ women, which may indicate that they were aware of how to protect against the transmission of HIV. However, the awareness of being infected by STI others than HIV did not affect sexual risk behaviour among the women. Our data show that STIs still constitute an ignored medical issue with consequences for women.

\section{Prevalence and incidence of STIs and RTIs differed between HIV- and HIV+ women}

The prevalence of STIs/RTIs at baseline was higher in HIVwomen than in HIV+ women; however, at the follow-up at 9 months, no differences in prevalence between HIV- and HIV+ women were observed. The differences in prevalence at baseline and 9 months in HIV+ women were to a large extent dependent on a lower prevalence of BV and syphilis at baseline compared 


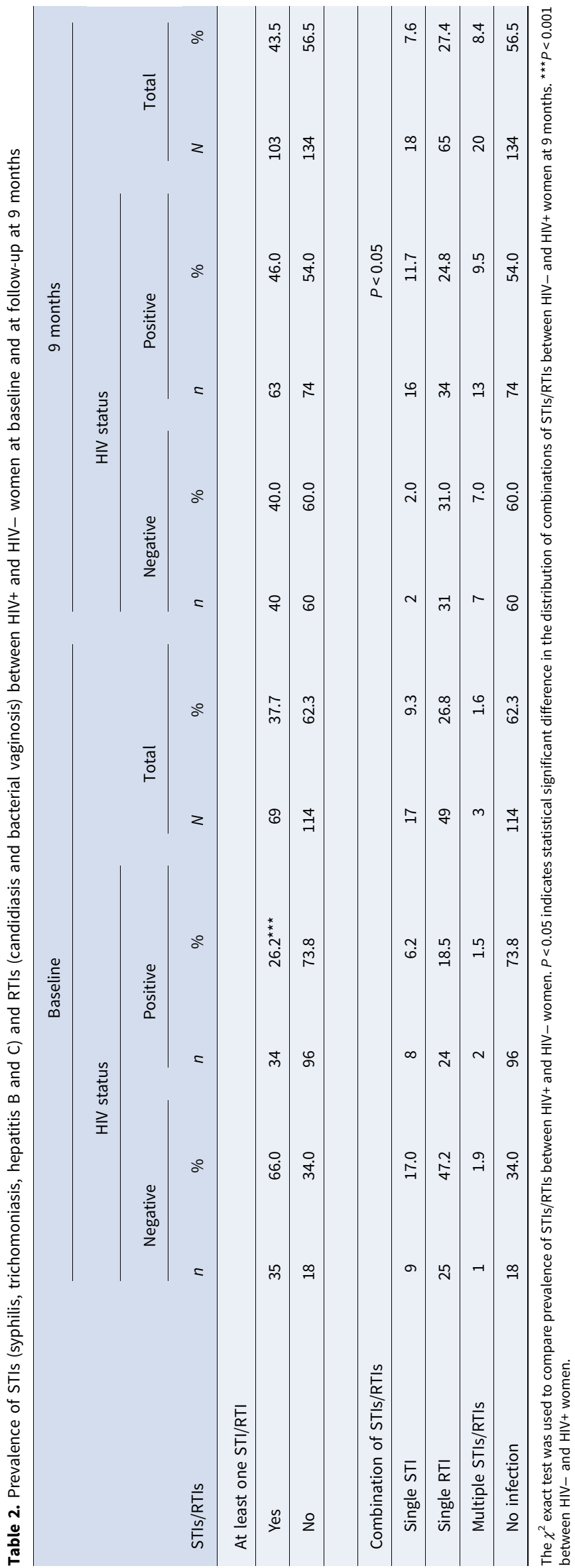

with follow-up at 9 months. Even though all tests were performed with the same technique and at the same laboratory, technical issues with the analyses at baseline could be an explanation to the discrepancies in prevalence. The current study shows that syphilis was more common among HIV+ women than among HIV- women in accordance with previous studies [13, 19-21]. Persistent candidiasis (present at baseline and at 9 months) was more common in HIV- women than in HIV+ women. One contributing factor was that HIV+ women were on a regular basis in contact with the HIV clinic, which resulted in that more HIV+ women than HIV- women were treated for candidiasis and for other RTIs/STIs.

\section{Regular use of condoms but still many STIs in HIV+ women}

Similar to previous studies in Africa [22], we showed that most of HIV+ women opted for sexual abstinence, while those who engaged in sexual activity claimed using condom. However, our study shows that 30 out of 32 cases of all infections observed at 9 months were new and were contracted during the follow-up period and that $82 \%$ were found among HIV+ women. The apparent paradox of high rate of condom use in the HIV+ group and the high rate of new infections has previously been demonstrated in several national surveys conducted in African countries [23, 24]. These studies showed that women use condoms less consistently than men depending on religious beliefs and to the inability for women to negotiate condom use with their partners. The social desirability bias among HIV+ women may possibly have contributed to the observed paradox of high degree of condom use and high degree of STIs/RTIs in our study.

\section{Sexual behaviour was not affected by STI or marital status}

Our data show that neither marital status nor being diagnosed with STIs at baseline seemed to change women's sexual behaviour. HIV-positive status alone was found to be associated with an acceptable level of condom use as it has been shown in other studies [25]. In our study, we show a low condom use among HIV- women despite that consistent condom use protects against HIV and other STIs [26]. The low percentage using condoms in people who are HIV-negative or not aware of HIV status is consistent with previous studies $[27,28]$. Low use of condoms has also been reported in married and partnered people in sub-Saharan Africa [29]. Studies show that condom use has decreased, while the use of injectable contraceptives has increased as a contraceptive method among women in Rwanda and Uganda [30]. This may as a consequence lead to an increased exposure to STIs including HIV. In the early HAART era, it was reported that people who were tested negative for HIV did not change their sexual risk behaviour compared with untested persons. This could depend on a reinforcement of previous sexual risk behaviour and increased risk behaviour [31]. Similar observations have been shown in other studies from sub-Saharan Africa [32]. Married women may also be at a higher risk of exposure to HIV than married men, since studies from Zambia show that unprotected sex is more common among couples where the husband is HIV-negative and the woman is HIV-positive than in the reverse situation of HIV status [33]. Besides access to health care, some of the barriers preventing African women to seek treatment for genital infections have been extensively studied, i.e. the asymptomatic characteristics of many of genital infections, the way in which women perceive symptoms as not seriously enough to 
Table 3. Prevalent infections at baseline, incident infections (absent at baseline and present at 9 months), persistent infections (present at baseline and 9 months) and prevalent infections at 9 months among HIV+ and HIV- women

\begin{tabular}{|c|c|c|c|c|c|c|c|c|c|c|c|c|}
\hline \multirow{3}{*}{$\begin{array}{l}\text { STIs/RTIs in } \\
\text { Rwandan women }\end{array}$} & \multicolumn{6}{|c|}{ Prevalence of infections at baseline } & \multicolumn{6}{|c|}{$\begin{array}{l}\text { Infections absent at baseline and present at } 9 \text { months } \\
\text { (incident infections) }\end{array}$} \\
\hline & \multicolumn{2}{|c|}{ HIV- } & \multicolumn{2}{|c|}{ HIV+ } & \multicolumn{2}{|c|}{ Total } & \multicolumn{2}{|c|}{ HIV- } & \multicolumn{2}{|c|}{ HIV+ } & \multicolumn{2}{|c|}{ Total } \\
\hline & $n$ & $\%$ & $n$ & $\%$ & N & $\%$ & $n$ & $\%$ & $n$ & $\%$ & N & $\%$ \\
\hline \multicolumn{13}{|l|}{ Hepatitis B } \\
\hline \multicolumn{13}{|l|}{ Hepatitis C } \\
\hline Positive & 0 & 0.0 & 4 & 3.1 & 4 & 2.6 & 0 & 0.0 & 6 & 4.8 & 6 & 4.1 \\
\hline Negative & 23 & 100.0 & 125 & 96.9 & 148 & 97.4 & 23 & 100.0 & 119 & 95.2 & 142 & 95.9 \\
\hline \multicolumn{13}{|l|}{ Trichomoniasis } \\
\hline Positive & 1 & 1.4 & 1 & .8 & 2 & 1.0 & 0 & 0.0 & 14 & $10.9^{\star \star}$ & 14 & 7.1 \\
\hline Negative & 68 & 98.6 & 128 & 99.2 & 196 & 99.0 & 68 & 100.0 & 114 & 89.1 & 182 & 92.9 \\
\hline \multicolumn{13}{|l|}{ Candidiasis } \\
\hline Positive & 12 & 12.0 & 13 & 9.5 & 25 & 10.5 & 18 & 20.5 & 19 & 15.3 & 37 & 17.5 \\
\hline Negative & 88 & 88.0 & 124 & 90.5 & 212 & 89.5 & 70 & 79.5 & 105 & 84.7 & 175 & 82.5 \\
\hline \multicolumn{13}{|l|}{ Bacterial vaginosis } \\
\hline Positive & 15 & 15.0 & 12 & 8.8 & 27 & 11.4 & 12 & 14.1 & 23 & 18.4 & 35 & 16.7 \\
\hline \multirow[t]{2}{*}{ Negative } & 85 & 85.0 & 125 & 91.2 & 210 & 88.6 & 73 & 85.9 & 102 & 81.6 & 175 & 83.3 \\
\hline & \multicolumn{6}{|c|}{ Infections present at baseline and 9 months (persistent infections) } & \multicolumn{6}{|c|}{ Prevalence of infections at 9 months } \\
\hline Hepatitis C & & & & & & & & & & & & \\
\hline Positive & 0 & 0.0 & 0 & 0.0 & 0 & 0.0 & 0 & 0.0 & 6 & 4.7 & 6 & 3.9 \\
\hline Negative & 0 & 0.0 & 4 & 100.0 & 4 & 100.0 & 23 & 100.0 & 123 & 95.3 & 146 & 96.1 \\
\hline Trichomoniasis & & & & & & & & & & & & \\
\hline Positive & 2 & 40.0 & 1 & 25.0 & 3 & 33.3 & 3 & 3.0 & 5 & 3.6 & 8 & 3.4 \\
\hline Negative & 3 & 60.0 & 3 & 75.0 & 6 & 66.7 & 96 & 97.0 & 132 & 96.4 & 228 & 96.6 \\
\hline Syphilis & & & & & & & & & & & & \\
\hline Positive & 0 & 0.0 & 0 & 0.0 & 0 & 0.0 & 0 & 0.0 & 14 & $10.9^{\star \star}$ & 14 & 7.1 \\
\hline Negative & 1 & 100.0 & 1 & 100.0 & 2 & 100.0 & 69 & 100.0 & 115 & 89.1 & 184 & 92.9 \\
\hline Candidiasis & & & & & & & & & & & & \\
\hline Positive & 6 & 50.0 & 1 & $7.7^{\star}$ & 7 & 28.0 & 24 & 24.0 & 20 & 14.6 & 44 & 18.6 \\
\hline Negative & 6 & 50.0 & 12 & 92.3 & 18 & 72.0 & 76 & 76.0 & 117 & 85.4 & 193 & 81.4 \\
\hline Bacterial vaginosis & & & & & & & & & & & & \\
\hline Positive & 6 & 40.0 & 8 & 66.7 & 14 & 51.9 & 18 & 18.0 & 31 & 22.6 & 49 & 20.7 \\
\hline Negative & 9 & 60.0 & 4 & 33.3 & 13 & 48.1 & 82 & 82.0 & 106 & 77.4 & 188 & 79.3 \\
\hline
\end{tabular}

For persistent infections, positive means persistent infection while negative means cleared (either after treatment or naturally) infection at 9 months. ${ }^{\star} P<0.05$ and ${ }^{\star \star} P<0.01$ between HIVand HIV+ women with the Fisher's exact test. 


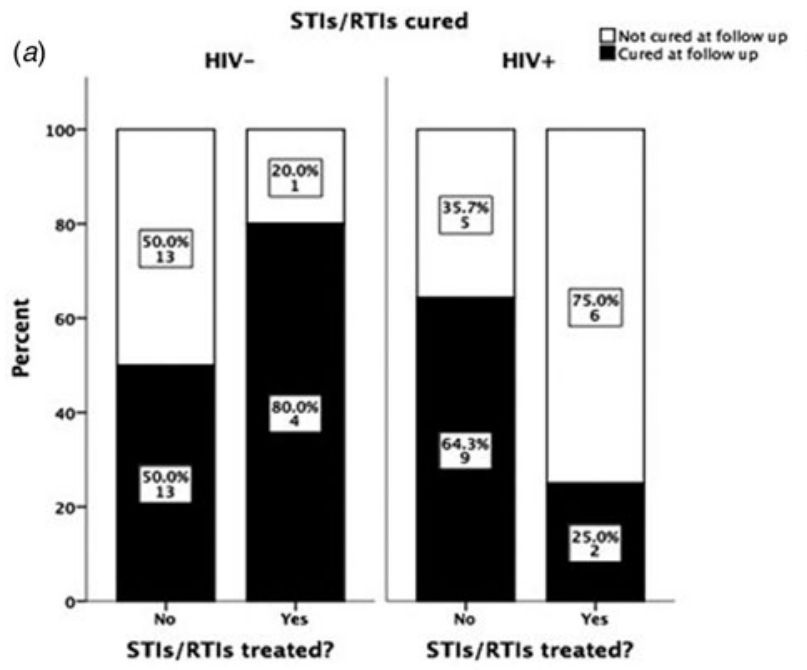

$\begin{array}{ll}\text { (b) } & \square \text { HIV- } \\ & \text { HIV+ }\end{array}$

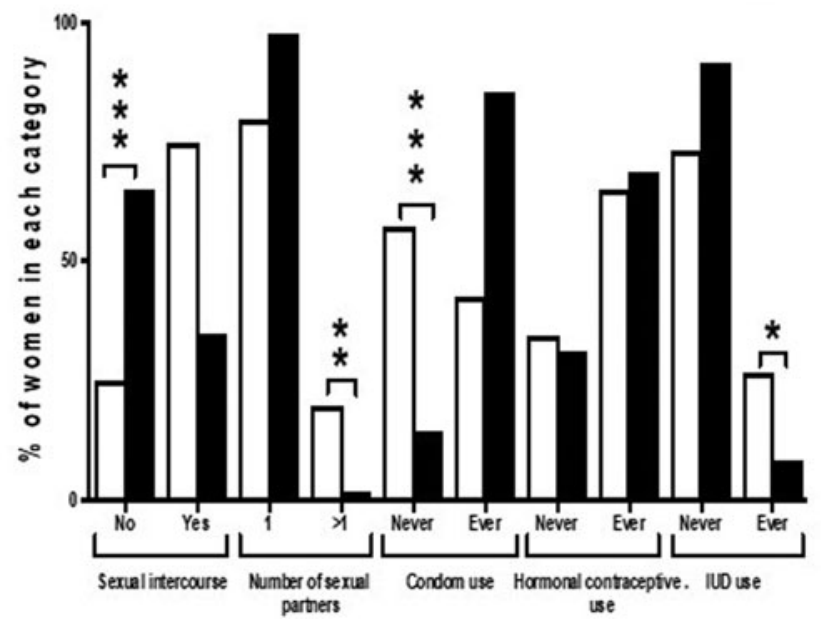

Fig. 1. Treatment-seeking behaviour and curing rates among HIV- and HIV+ women positive for STIs/RTIs (a). The graph displays women who had a positive test results for at least one of the studied STIs/RTIs ( $n=53$ ). 'Cured' indicates women where all infections that were present at baseline were cured at follow-up. The Fisher's exact test was used to compare behaviour between HIV+ and HIV- women. Sexual behavioural characteristics during 9 months of follow-up among HIV+ and HIV- women (b). The $\chi^{2}$ test was used to compare behaviour between HIV+ and HIV- women. ${ }^{\star} P<0.05,{ }^{\star \star} P<0.01$ and ${ }^{\star \star \star} P<0.001$.

(a)

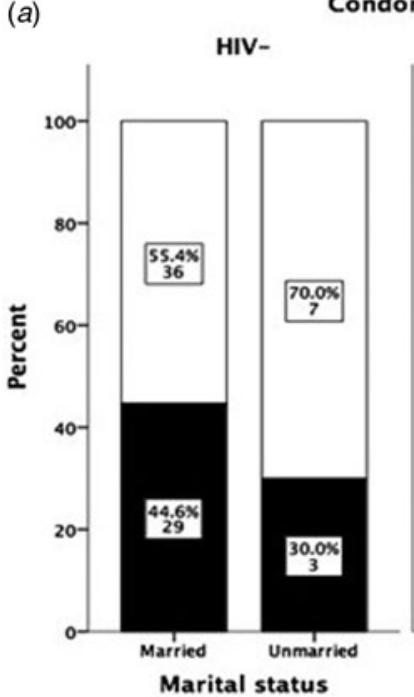

Condom use

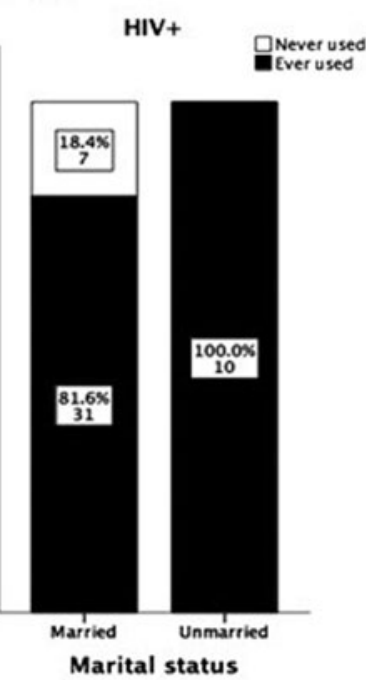

(b)

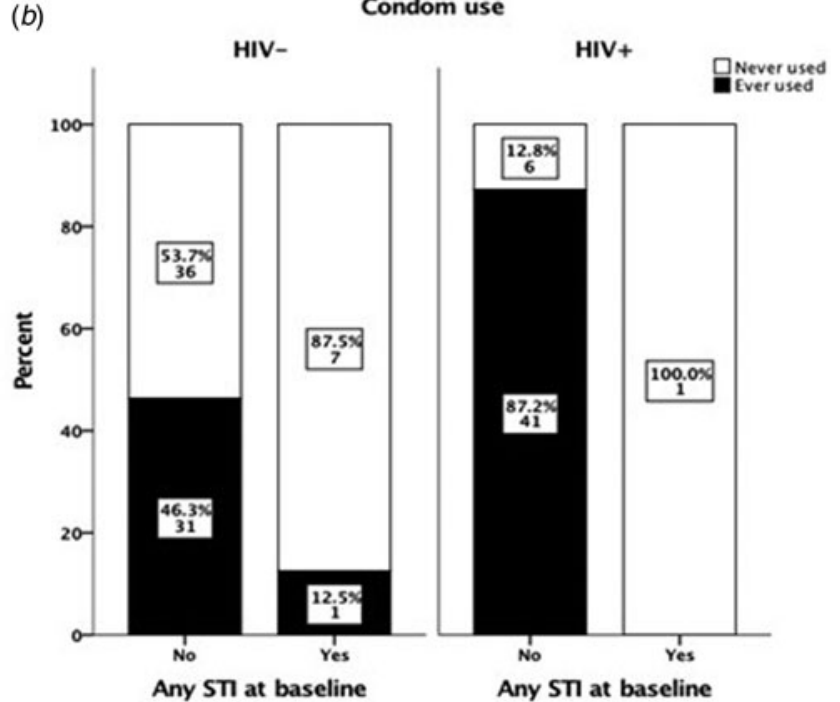

Fig. 2. Effect of marital status and HIV status on condom use during 9 months of follow-up (a). The data are based only on results from women who claimed to have had sexual intercourse during the follow-up period. Effect of STI test results at baseline on condom use among HIV- and HIV+ women (b). The data are based only on results from women who claimed to have had sexual intercourse during the follow-up period and to whom the results on the STI test done at baseline were communicated. The $\chi^{2}$ exact test was used to compare differences in condom use among groups.

seek treatment, self-medication among women and the stigma attached to seek treatment for STIs [34]. As a consequence to this, complications may arise, e.g. leaving BV untreated may lead to preterm labour and postpartum endometritis [35]. Well known is also the risk to develop neurological complications if syphilis is left untreated [36].

\section{Limitations of study}

We have to state some of the limitations of our study. First, we had a low number of individual STI and RTI and therefore our study does not reveal how individual STI and RTI affected sexual behaviour. Second, we did not test for gonorrhoea, chlamydia and herpes, which are common in many countries in sub-Saharan Africa. Third, sexual behaviour is a sensitive topic especially for Rwandan women in their culture. Thus, it may be possible, in face-to-face interview that the social desirability bias as mentioned contributed to our findings and to an overestimation of factors such as the use of condoms. The sensitive topic is also reflected by the fact that $10 \mathrm{HIV}+$ women claimed not having sex but still contracted syphilis during the follow-up. To get as reliable answers as possible, the interview was therefore conducted by a medical doctor or a nurse at the gynaecology departments. At the HIV clinic at CHUK, where gynaecological examinations are 
not normally performed, the interview was conducted by a physician or by a nurse in charge of counselling, thus in whom women felt normally confident. Fourth, half of the studied cohort consisted of HPV-positive women and/or with cytological changes and that it is well established that HPV positivity correlates with high-risk sexual behaviour [37], and therefore we cannot absolutely extrapolate our findings to the general female Rwandan population. Fifth, we do not have data on sexual partners and if women were living with HIV discordant or concordant partners affecting sexual behaviour among women. Despite the possible limitations, our study demonstrated that STIs/RTIs continue to be an important public health issue that needs to be reconsidered.

In conclusion, our study shows a high prevalence of STIs and RTIs among Rwandan women where sexual risk behaviour is changed upon HIV positivity awareness but not necessarily upon the awareness of being infected by other STIs. Our data highlight the importance of public education regarding condom use to protect against STIs in an era when HIV no longer is a death sentence.

Supplementary material. The supplementary material for this article can be found at https://doi.org/10.1017/S0950268818003023.

Acknowledgement. The authors thank all research women and research assistants for their contribution to the success of the study.

Financial support. This study was supported by the Swedish International Development Cooperation Agency (Sida).

Conflict of interest. None.

Author ORCIDs. (D) D. Giglio 0000-0002-1349-9336

\section{References}

1. Mudau M et al. (2018) High prevalence of asymptomatic sexually transmitted infections among human immunodeficiency virus-infected pregnant women in a low-income South African community. International Journal of STD \& AIDS 29, 324-333.

2. Maina AN, Kimani J and Anzala O (2016) Prevalence and risk factors of three curable sexually transmitted infections among women in Nairobi, Kenya. BMC Research Notes 9, 193.

3. Naidoo S et al. (2014) High prevalence and incidence of sexually transmitted infections among women living in Kwazulu-Natal, South Africa. AIDS Research and Therapy 11, 31.

4. Buvé A et al. (2014) The vaginal microbiota and susceptibility to HIV. AIDS (London, England) 28, 2333-2344.

5. Williamson A-L (2015) The interaction between human immunodeficiency virus and human papillomaviruses in heterosexuals in Africa. Journal of Clinical Medicine 4, 579-592.

6. McClelland RS et al. (2007) Infection with Trichomonas vaginalis increases the risk of HIV-1 acquisition. The Journal of Infectious Diseases 195, 698702.

7. Ward HRM (2010) Contribution of sexually transmitted infections to the sexual transmission of HIV. Current Opinion in Hiv and Aids 5, 305-310.

8. Clifford GM et al. (2016) Effect of HIV infection on human papillomavirus types causing invasive cervical cancer in Africa. JAIDS Journal of Acquired Immune Deficiency Syndromes 73, 332-339.

9. Forbi J et al. (2009) Seroprevalence of syphilis among a cohort of HIV-infected subjects in North Central Nigeria. Journal of Health, Population, and Nutrition 27, 704-706.

10. McClelland RS et al. (2005) Contribution of HIV-1 infection to acquisition of sexually transmitted disease: a 10 -year prospective study. The Journal of Infectious Diseases 191, 333-338.
11. Augenbraun M et al. (1995) Increased genital shedding of herpes simplex virus type 2 in HIV-seropositive women. Annals of Internal Medicine 123, 845-847.

12. Bertini M (2017) Bacterial vaginosis and sexually transmitted diseases: relationship and management. In Serdaroglu $S$ and Kutlubay $Z$ (eds), Fundamentals of Sexually Transmitted Infections. Rijeka: InTech, pp. Ch. 05.

13. Mutagoma $\mathbf{M}$ et al. (2016) The prevalence of syphilis infection and its associated factors in the general population of Rwanda: a national household-based survey. Journal of Sexually Transmitted Diseases 2016, 4980417.

14. Mukanyangezi MF et al. (2018) Screening for human papillomavirus, cervical cytological abnormalities and associated risk factors in HIV-positive and HIV-negative women in Rwanda. HIV Medicine 19, 152-166.

15. Torres-Rueda S et al. (2016) HPV vaccine introduction in Rwanda: impacts on the broader health system. Sexual \& Reproductive Healthcare 7, 46-51.

16. Muñoz N et al. (2003) Epidemiologic classification of human papillomavirus types associated with cervical cancer. New England Journal of Medicine 348, 518-527.

17. Schmitt M et al. (2008) Homogeneous amplification of genital human alpha papillomaviruses by PCR using novel broad-spectrum GP5+ and GP6+ primers. Journal of Clinical Microbiology 46, 1050-1059.

18. Nugent RP, Krohn MA and Hillier SL (1991) Reliability of diagnosing bacterial vaginosis is improved by a standardized method of gram stain interpretation. Journal of Clinical Microbiology 29, 297-301.

19. Jespers V et al. (2014) Prevalence and correlates of bacterial vaginosis in different sub-populations of women in sub-Saharan Africa: a crosssectional study. PLoS ONE 9, e109670.

20. Dionne-Odom J et al. (2013) Syphilis treatment response among HIV-discordant couples in Zambia and Rwanda. Journal of Biochemistry and Molecular Biology 56, 1829-1837.

21. Mutagoma M et al. (2017) Ten-year trends of syphilis in sero-surveillance of pregnant women in Rwanda and correlates of syphilis-HIV co-infection. International Journal of STD \& AIDS 28, 45-53.

22. Dokubo EK et al. (2014) Awareness of HIV status, prevention knowledge and condom use among people living with HIV in Mozambique. PLoS ONE 9, e106760.

23. Risher K et al. (2016) Antiretroviral treatment and sexual risk behavior in South Africa. AIDS and Behavior 20, 710-716.

24. Ali Abdulai M et al. (2017) The risk of sexually transmitted infection and Its influence on condom use among pregnant women in the Kintampo North Municipality of Ghana. Journal of Sexually Transmitted Diseases 2017, 12.

25. O'Campo P et al. (1992) Prior episode of sexually transmitted disease and subsequent sexual risk-reduction practices. A need for improved risk-reduction interventions. Sexually Transmitted Diseases 19, 326-330.

26. Ahmed S et al. (2001) HIV incidence and sexually transmitted disease prevalence associated with condom use: a population study in Rakai, Uganda. AIDS (London, England) 15, 2171-2179.

27. Rugigana E, Birungi $\mathbf{F}$ and Nzayirambaho M (2015) HIV knowledge and risky sexual behavior among men in Rwanda. Pan African Medical Journal 22, 380 .

28. Gautam R et al. (2015) Correlates of the molecular vaginal microbiota composition of African women. BMC Infectious Diseases 15, 86.

29. Shi CF, Li M and Dushoff J (2017) Evidence that promotion of male circumcision did not lead to sexual risk compensation in prioritized sub-Saharan countries. PLOS ONE 12, e0175928.

30. Dennis ML et al. (2017) Pathways to increased coverage: an analysis of time trends in contraceptive need and use among adolescents and young women in Kenya, Rwanda, Tanzania, and Uganda. Reproductive Health 14, 130.

31. Weinhardt LS et al. (1999) Effects of HIV counseling and testing on sexual risk behavior: a meta-analytic review of published research, 19851997. American Journal of Public Health 89, 1397-1405.

32. Rosenberg NE et al. (2017) The impact of couple HIV testing and counseling on consistent condom use among pregnant women and their male partners: an observational study. JAIDS Journal of Acquired Immune Deficiency Syndromes 75, 417-425. 
33. Wall KM et al. (2017) Optimizing prevention of HIV and unplanned pregnancy in discordant African couples. J Womens Health (Larchmt) 26, 900-910.

34. Nasirian M et al. (2015) Care seeking patterns of STIs-associated symptoms in Iran: findings of a population-based survey. International Journal of Health Policy and Management 5, 5-11.

35. Jalagadugula Venkata Narasimha R and Jalagadugula C (2017) The association of bacterial vaginosis with adverse pregnancy outcomes. Journal of Evidence Based Medicine and Healthcare 4, 30403042

36. Clark EG and Danbolt N (1955) The Oslo study of the natural history of untreated syphilis: an epidemiologic investigation based on a restudy of the Boeck-Bruusgaard material a review and appraisal. Journal of Chronic Diseases 2, 311-344.

37. Lenselink CH et al. (2008) Sexual behaviour and HPV infections in 18 to 29 year old women in the pre-vaccine era in the Netherlands. PLOS ONE 3, e3743. 